\title{
HOMOGENEOUS CONTINUA IN EUCLIDEAN $(n+1)$-SPACE WHICH CONTAIN AN $n$-CUBE ARE LOCALLY CONNECTED
}

\author{
JANUSZ R. PRAJS
}

\begin{abstract}
We prove that each homogeneous continuum which topologically contains an $n$-dimensional unit cube and lies in $(n+1)$-dimensional Euclidean space is locally connected.
\end{abstract}

Introduction. Although we still do not have any complete classification of homogeneous plane continua today, there are many significant results concerning homogeneous continua in the planar case. It is interesting to consider which of these results may be naturally extended to higher dimensional cases. In this paper we give an example of such an extension. In $1924 \mathrm{~S}$. Mazurkiewicz [7] proved that a simple closed curve is the only homogeneous plane continuum which is nondegenerate and locally connected. Using this result, R. H. Bing showed in 1959 [1] that a simple closed curve is the only homogeneous plane continuum that contains an arc. All that he had to do to show this (and that he did) was to prove that each homogeneous plane continuum which contains an arc is locally connected. Here we extend the last statement to all finite dimensional cases.

MAIN THEOREM. If a homogeneous continuum contains a set homeomorphic to the $n$-dimensional unit cube and lies in $(n+1)$-dimensional Euclidean space, then it is locally connected.

It is very natural to ask whether the assumptions of this theorem imply that the considered continuum is an $n$-manifold. Recently the author has answered this question in the affirmative. This result, which makes heavy use of the Main Theorem, will be presented in a future paper.

The general method of the proof of the Main Theorem is similar to that of Bing's theorem, i.e. we suppose the existence of a continuum not satisfying this theorem and find a sequence of properties of it. Finally we get two properties such that one contradicts the other. However, only Property 1 here is similar to Property 4 in $[\mathbf{1}$, p. 212], and the particular method of proof here differs much from Bing's. There is also another important difference from the Bing method-we use many times the so-called $\varepsilon$-push property of homogeneous metric continua (see Lemma 4 in [2, p. 37]) which is a corollary to the well-known Effros theorem, and which was not known to Bing in 1959.

The author wishes to express profound thanks to Professor J. J. Charatonik for all his help in completing this work.

Received by the editors October 15, 1986 and, in revised form, April 10, 1987.

1980 Mathematics Subject Classification (1985 Revision). Primary 54F 20; Secondary 54C25.

Key words and phrases. Continuum, embedding, Euclidean space, homogeneity, local connectedness. 
Throughout the paper the symbol $I$ denotes the unit segment $[0,1], E^{n}$ is $n$ dimensional Euclidean space, $\rho(x, y)$ is the usual Euclidean distance between points $x$ and $y$ of a Euclidean space. We write $\operatorname{Lim} A_{n}=A$ when $\left\{A_{n}\right\}$ is a sequence of closed sets converging to a set $A$ (with respect to the Hausdorff distance). Further, $B(x, \varepsilon)=\left\{y \in E^{n}: \rho(x, y)<\varepsilon\right\}$ and $\rho(A, B)=\inf \{\rho(a, b): a \in A$ and $b \in B\}$, where $\varepsilon>0$ and $A, B \subset E^{n}$. Also,

$$
S_{n}=\left\{x \in E^{n+1}: \rho(x, \mathbf{0})=1\right\} \subset B_{n+1}=\left\{x \in E^{n+1}: \rho(x, \mathbf{0}) \leq 1\right\}
$$

where $\mathbf{0}=(0, \ldots, 0) \in E^{n+1}$. If a set $A$ satisfies $A \underset{\text { top }}{=} I^{n}$, its combinatorial boundary is denoted by $\partial A$. The symbol $x y$ denotes an arc with end points $x$ and $y$. For two points $p, q \in E^{n}$, the symbol $\overline{p q}$ denotes the segment between these points. A set $T$ is called an $n$-dimensional simple triod if it is the union $T=A \cup x y$ with $A \underset{\text { top }}{=} I^{n}$ and $A \cap x y=\{x\} \subset A \backslash \partial A$. A point $x$ is said to be accessible from a set $Y$ provided there exists an arc $x y$ such that $x y \backslash\{x\} \subset Y$. The notation $f: X \rightarrow Y$ always means that $f$ is a continuous mapping from a space $X$ into a space $Y$. A map $f: A \rightarrow X$, for $A \subset X$, is called an $\varepsilon$-mapping if $\rho(a, f(a))<\varepsilon$ for each $a \in A$. A space is said to be a manifold if its topology has a basis composed of open sets homeomorphic to the same Euclidean space.

Preliminaries. We will give or remind the reader of a few facts which we need to prove the Main Theorem. We begin with a well-known theorem of Borsuk.

1. THEOREM [4, THEOREM 7, p. 495]. If two compact sets $A, B \subset E^{n}$ are homeomorphic, then the numbers of components of the sets $E^{n} \backslash A$ and $E^{n} \backslash B$ are equal.

A set $A \subset X$ separates a space $X$ provided $X \backslash A$ is not connected. It separates $X$ between points $p, q \in X$ provided $p$ and $q$ lie in distinct components of $X \backslash A$. The next theorem is an immediate consequence of the two following results: [4, Theorem 2, p. 473 and 4, Theorem 8, p. 258].

2. THEOREM. If a compact set $F \subset S_{n} \backslash\{p, q\}$ separates $S_{n}$ between $p$ and $q$, with $p, q \in S_{n}$, then each set which is obtained from $F$ by a deformation in $S_{n} \backslash\{p, q\}$ separates $S_{n}$ between $p$ and $q$.

3. Proposition. If a set $A \subset E^{n+1}$ is homeomorphic to $I^{n}, p \in E^{n+1}$ and $\rho(p, \partial A) \geq \varepsilon>\rho(p, A)$, then for every component $B$ of $B(p, \varepsilon) \cap A$ the set $B(p, \varepsilon) \backslash B$ has exactly two components.

PROOF. Let a mapping $f: B \rightarrow S_{n}$ be an embedding. Define two functions $t: S_{n} \rightarrow E^{1}$ and $h: S_{n} \rightarrow h\left(S_{n}\right) \subset E^{n+1}$ by

$$
t(x)= \begin{cases}\rho\left(x, S_{n} \backslash f(B)\right) & \text { if } x \in f(B), \\ 0 & \text { otherwise, }\end{cases}
$$

and $h(x)=(1+t(x)) \cdot x$. Then $h$ is a homeomorphism. Consider the quotient space $E^{n+1} / B_{n+1}$ with the quotient map $\pi: E^{n+1} \rightarrow E^{n+1} / B_{n+1}$. The proofs of the two following facts are left to the reader.

(1) The set $E^{n+1} \backslash\left(S_{n} \cup h\left(S_{n}\right)\right)$ has exactly three components.

(2) The set $E^{n+1} / B_{n+1} \backslash \pi\left(S_{n} \cup h\left(S_{n}\right)\right)$ has exactly two components. 
Let $q \in \operatorname{bd} B(p, \varepsilon) \backslash \operatorname{cl} B$ and $\rho(q, \operatorname{cl} B)>\delta>0$. Then

$$
Y=\operatorname{bd} B(p, \varepsilon) \backslash B(q, \delta) \underset{\text { top }}{=} I^{n} .
$$

Consider the quotient space $E^{n+1} / Y$ with the quotient map $\pi_{1}: E^{n+1} \rightarrow E^{n+1} / Y$. Then we have

$$
E^{n+1} \underset{\text { top }}{=} E^{n+1} / B_{n+1} \underset{\text { top }}{=} E^{n+1} / Y \text { and } \pi\left(S_{n} \cup h\left(S_{n}\right)\right) \underset{\text { top }}{=} \pi_{1}(\mathrm{cl} B)
$$

(the second of these equalities is a consequence of the fact that both considered sets are one-point compactifications of sets homeomorphic to $B$ ); thus it follows from Theorem 1 that the set $E^{n+1} / Y \backslash \pi_{1}(\mathrm{cl} B)$ has exactly two components. But $\left.\pi_{1}\right|_{B(p, \varepsilon)}$ is a homeomorphism and clearly the set $B(p, \varepsilon) \backslash B \underset{\text { top }}{=} \pi_{1}(B(p, \varepsilon) \backslash B)$ has exactly two components too.

4. Proposition. If a locally connected closed subset $A$ of $E^{n}$ separates $E^{n}$ between two points $p, q \in E^{n}$, then there is a component of $A$ that also does.

ProOF. Suppose no component of $A$ separates $E^{n}$ between $p$ and $q$. Consider a one-point compactification $E^{n} \cup\{z\} \underset{\text { top }}{=} S_{n}$ of $E^{n}$ with the remainder $z$. By the local connectedness of $A$ only a finite number of components of $A$ intersect the segment $\overline{p q}$ and the union of all components not intersecting this segment is closed in $E^{n}$. Let $B_{0}$ be the closure (in $E^{n} \cup\{z\}$ ) of this union and $B_{1}, \ldots, B_{m}$ be the closures (in $E^{n} \cup\{z\}$ ) of the components intersecting $\overline{p q}$. Of course, no set $B_{i}$ for $i \in\{0,1, \ldots, m\}$ separates $E^{n} \cup\{z\}$ between $p$ and $q$. Obviously, the claim that the union $B_{0} \cup B_{1} \cup \cdots \cup B_{m}$ does not separate $E^{n} \cup\{z\}$ between $p$ and $q$ completes the proof. Indeed, if $n>2$, we have $\operatorname{dim}\left(B_{i} \cap B_{j}\right) \leq \operatorname{dim}\{z\}=0 \leq n-3$ for $i, j \in\{0,1, \ldots, m\}$ and $i \neq j$. Applying $m$ times Theorem 11 of $[\mathbf{4}, \mathbf{p} .471]$, the claim is obtained. To prove the case $n=2$ use the same theorem and footnote (1) of $[4$, p. 471]. The case $n=1$ is trivial.

REMARK. Note that the word "component" may be replaced by "arc component" in Propositions 3 and 4 . Observe that in Proposition 4 the open balls in $E^{n}$ may be considered instead of $E^{n}$.

5. Proposition. Let a closed subset $C$ of a ball $B(c, \varepsilon) \subset E^{n}$ separate this ball between two points $p, q \in B(c, \varepsilon) \backslash C$. Then for every $\delta>0$ such that $\delta<$ $\rho\left(\{p, q\}, C \cup\left(E^{n} \backslash B(c, \varepsilon)\right)\right)$ and for every $\delta$-mapping $f: C \rightarrow E^{n}$ the set $f(C)$ separates the ball $B(c, \varepsilon-\delta)$ between $p$ and $q$.

Proof. Suppose $f(C)$ does not separate $B(c, \varepsilon-\delta)$ between $p$ and $q$. Let a sequence $\left\{x_{n}\right\}$ of points of $f(C) \cap B(c, \varepsilon-\delta)$ converge to a point $x \in B(c, \varepsilon-\delta)$. Then $\rho\left(x_{n}\right.$, bd $\left.B(c, \varepsilon)\right)>\delta+\delta_{1}$ for some $\delta_{1}>0$ and every $x_{n}$. Thus $f^{-1}\left(\left\{x_{1}, x_{2}, \ldots\right\}\right) \subset$ $C \cap \operatorname{cl} B\left(c, \varepsilon-\delta_{1}\right)$. The last set is compact, so $x \in f(C)$. Hence the set $f(C) \cap$ $B(c, \varepsilon-\delta)$ is closed in $B(c, \varepsilon-\delta)$. Thus there is an arc $p q$ in $B\left(c, \varepsilon-\delta-\delta_{2}\right) \backslash f(C)$ for some positive number $\delta_{2}<\rho\left(\{p, q\}, E^{n} \backslash B(c, \varepsilon)\right)-\delta$. So $f(C)$ does not separate $B\left(c, \varepsilon-\delta-\delta_{2}\right)$ between $p$ and $q$. Consider the mapping $g: C \rightarrow B(\mathbf{0}, \delta)$, where $\mathbf{0}=(0, \ldots, 0) \in E^{n}$, defined by $g(x)=f(x)-x$. By the Tietze theorem this mapping may be extended to a mapping $g^{*}: C_{1} \rightarrow B(\mathbf{0}, \delta)$, where $C_{1}=C \cup\{x \in$ $\left.E^{n}: \rho(c, x)=\varepsilon-\delta_{2}\right\}$. Thus we get the extension $f^{*}: C_{1} \rightarrow E^{n}$ of the mapping $f$, defined by $f^{*}(x)=x+g^{*}(x)$. Observe that $f^{*}$ is a $\delta$-mapping. Consider the 
deformation $G(x, t)=x+t \cdot g^{*}(x)$. We have $G: C_{1} \times I \rightarrow E^{n} \backslash\{p, q\}$. Note that the set $C_{1}=G\left(C_{1} \times\{0\}\right)$ separates $E^{n}$ between $p$ and $q$. By a simple application of Theorem 2 we see that the set $f^{*}\left(C_{1}\right)=G\left(C_{1} \times\{1\}\right)$ separates $E^{n}$ between $p$ and $q$. But no point of $f^{*}\left(C_{1} \backslash C\right)$ lies in $B\left(c, \varepsilon-\delta-\delta_{2}\right)$. Therefore $f^{*}(C)=f(C)$ separates $B\left(c, \varepsilon-\delta-\delta_{2}\right)$ between $p$ and $q$, a contradiction.

6. Proposition. If $A_{0}, A_{1}, A_{2}, \ldots$ are closed subsets of $E^{n}$ such that there are $\varepsilon_{k}$-mappings $f_{k}: A_{0} \rightarrow A_{k}$ with $\lim \varepsilon_{k}=0$, then for each ball $B(c, \varepsilon) \subset E^{n}$ with two points $p, q \in B(c, \varepsilon) \backslash A_{0}$ the set $A_{0}$ separates $B(c, \varepsilon)$ between $p$ and $q$ if and only if for every $\delta$ satisfying $\max \{\rho(p, c), \rho(q, c)\}<\delta<\varepsilon$ almost all $A_{k}$ separate the ball $B(c, \delta)$ between $p$ and $q$.

ProOf. Let $A_{0}$ separate $B(c, \varepsilon)$ between $p$ and $q$. Then the fact that almost all $A_{k}$ separate $B(c, \delta)$ between $p$ and $q$ easily follows by Proposition 5 . Assume $A_{0}$ does not separate $B(c, \varepsilon)$ between $p$ and $q$. Then there are an arc $p q \subset B(c, \varepsilon) \backslash A_{0}$ and an integer $m_{0}$ such that for each $m>m_{0}$ we have $\rho\left(p q, A_{0}\right)>\varepsilon_{m}$. Put $\delta=\varepsilon-\rho(p q, \operatorname{bd} B(c, \varepsilon)) / 2$. It is clear that $A_{m}$ does not separate $B(c, \delta)$ between $p$ and $q$ for $m>m_{0}$.

7. Proposition. Let sets $A_{0}, A \subset E^{n+1}$ be homeomorphic to $I^{n}$ and a ball $B(c, \varepsilon) \subset E^{n+1}$ be such that $A_{0} \subset A \cap B(c, \varepsilon) \subset A \backslash \partial A$. Fix a point $p \in A_{0} \backslash \partial A_{0}$. Let $B$ be the arc component of $A \cap B(c, \varepsilon)$ containing $p$, and $U$ be a component of $B(c, \varepsilon) \backslash B$. If there is a sequence of $\varepsilon_{k}$-mappings $f_{k}: A_{0} \rightarrow f_{k}\left(A_{0}\right) \subset U$ such that $\lim \varepsilon_{k}=0$, then $p$ is not accessible from $U \backslash\left(f_{1}\left(A_{0}\right) \cup f_{2}\left(A_{0}\right) \cup \cdots\right)$.

PROOF. Let $p a$ be an arc with $p a \backslash\{p\} \subset U$. Denote by $V$ the other component of $B(c, \varepsilon) \backslash B$ (see Proposition 3). Let $\left\{a_{k}\right\},\left\{q_{k}\right\},\left\{z_{k}\right\}$ be sequences such that $a_{k} \in$ $p a \backslash\{p\}$ and $\lim a_{k}=p, q_{k} \in V$ and $\lim q_{k}=p, z_{k}$ is the first point of the segment $\overline{q_{k} a_{k}}$ which lies in $B$ (in the ordering from $q_{k}$ to $a_{k}$ ). Put $\delta=\rho\left(p, A \backslash A_{0}\right) / 2>0$ and let $B_{0}$ be the component of $A \cap B(p, \delta)=A_{0} \cap B(p, \delta)$ containing $p$. There is an integer $m$ such that $z_{m} \in B_{0}, q_{m} \in B(p, \delta)$ and $a_{m} p \subset B(p, \delta)$, where $a_{m} p \subset a p$. Since $B_{0}$ is also an arc component of $A_{0} \cap B(p, \delta)$, there is an arc $p z_{m} \subset B_{0}$. Put $a_{m} q_{m}=a_{m} p \cup p z_{m} \cup \overline{z_{m} q_{m}}$. $A_{0}$ separates $B(p, \delta)$ between $a_{m}$ and $q_{m}$; thus by Proposition 6 there exists an integer $i>0$ such that $a_{m} q_{m} \cap f_{i}\left(A_{0}\right) \neq \varnothing$. But $f_{i}\left(A_{0}\right) \cap\left(p z_{m} \cup \overline{z_{m} q_{m}}\right)=\varnothing$; hence $\varnothing \neq a_{m} p \cap f_{i}\left(A_{0}\right) \subset a p \cap f_{i}\left(A_{0}\right)$. Therefore the point $p$ is not accessible from $U \backslash\left(f_{1}\left(A_{0}\right) \cup f_{2}\left(A_{0}\right) \cup \cdots\right)$.

We end this part of the paper reminding the reader of two well-known results which are essential to the remaining part of the paper.

8. TheOREM [5, COROllary 2, p. 276]. Each Euclidean space $E^{n+1}$ does not contain uncountably many mutually exclusive $n$-dimensional simple triods.

9. THEOREM ( $\varepsilon$-PUSH PROPERTY [3, LEMMA 4, p. 37]). If $X$ is a homogeneous continuum and $\varepsilon>0$, then there exists $\delta>0$ such that for all $x, y \in X$ with $\rho(x, y)<\delta$ there is a homeomorphism $h: X \rightarrow X$ moving no point more than $\varepsilon$, such that $h(x)=y$.

Proof of the Main Theorem. Let $M$ be a homogeneous continuum lying in $E^{n+1}$ and containing a set homeomorphic to the $n$-dimensional unit cube $I^{n}$. Suppose $M$ is not locally connected. We will prove some properties of $M$ and find two of them such that one contradicts the other. Therefore we will infer that $M$ is locally connected. 


\section{PROPERTY 1. $M$ does not contain an n-dimensional simple triod.}

PROOF. It is known that a homogeneous continuum which is not locally connected contains an open set with uncountably many components (it can easily be proved by the reader either directly or with help of Theorem 2 in [1, p. 211]). If $M$ contained an $n$-dimensional simple triod, then by its homogeneity each component of an open subset with uncountably many components would contain such a triod, an impossibility by Theorem 8 .

PROPERTY 2. For every $\varepsilon>0$ there is $\delta>0$ such that for each $x \in M$ there exists a set $A$ homeomorphic to $I^{n}$ with $x \in A \subset B(x, \varepsilon) \cap M$ and $\rho(x, \partial A)>\delta$.

ProOF. Let $\varepsilon>0$. Of course, for each $x \in M$ there is a set $A \underset{\text { top }}{=} I^{n}$ with $x \in A \backslash \partial A \subset B(x, \varepsilon) \cap M$. Put $\sigma(x)=\sup \left\{\sigma>0:\right.$ there is $A \underset{\text { top }}{=} I^{n}$ with $x \in A \subset B(x, \varepsilon) \cap M$ and $\rho(x, \partial A)>\sigma\}$. Suppose the required $\delta$ does not exist. Then there is a convergent sequence $\left\{x_{m}\right\}$ of points in $M$ with $\lim \sigma\left(x_{m}\right)=0$. Put $x_{0}=\lim x_{m}$. Let $I^{n} \underset{\text { top }}{=} A_{0} \subset B\left(x_{0}, \varepsilon\right) \cap M, x_{0} \in A_{0} \backslash \partial A_{0}$ and put $\xi=\left(x_{0}, \partial A_{0}\right) / 2$. By Theorem 9 there are $\varepsilon_{m}$-homeomorphisms $h_{m}: M \rightarrow M$ with $h_{m}\left(x_{0}\right)=x_{m}$ and $\lim \varepsilon_{m}=0$. Considering the sets $h_{m}\left(A_{0}\right)$, we see that $\lim \inf \sigma\left(x_{m}\right)>\xi>0$, a contradiction.

The next property seems to be essential in our argument. As the reader will see, it will be employed very often. Bing in [1] made heavy use of the fact that his continuum $M$ contained no folded sequence of arcs converging to an arc (see Theorem 6 in [1, p. 220]). It may be observed that in case $n=1$ this fact is hidden in the next property, and this property is its virtual generalization, which is suitable for the proof of our theorem. To formulate this property, we define a number

$$
d(x, y)=\inf \{\operatorname{diam} A: A \text { is an arc and } x, y \in A \subset M\}
$$

for each pair of points $x, y$ of the same arc component of $M$.

PROPERTY 3. There exists $\varepsilon>0$ such that for every $\delta>0$ there is $\xi>0$ satisfying the following condition: if $x$ and $y$ are points of the same arc component of $M, d(x, y)<\varepsilon$ and $\rho(x, y)<\xi$, then $d(x, y)<\delta$.

PROOF. Let $\delta_{0}$ be a number guaranteed by Property 2 for the number $\varepsilon_{0}=$ $\operatorname{diam} M$ and put $\varepsilon=\delta_{0} / 2$. Suppose there exists $\delta>0$ such that for every positive integer $m$ there are points $x_{m}, y_{m}$ of the same arc component of $M$ fulfilling $d\left(x_{m}, y_{m}\right)<\varepsilon, \rho\left(x_{m}, y_{m}\right)<1 / m$ and $d\left(x_{m}, y_{m}\right)>\delta$. We may assume that the sequence $\left\{x_{m}\right\}$ converges to a point $x_{0} \in M$. By Theorem 9 there are $\varepsilon_{m^{-}}$ homeomorphisms $h_{m}: M \rightarrow M$ such that $h_{m}\left(x_{m}\right)=x_{0}$ and $\lim \varepsilon_{m}=0$. Let $A_{0} \subset M$ be a set homeomorphic to $I^{n}$ such that $x_{0} \in A_{0} \subset B\left(x_{0}, \varepsilon_{0}\right) \cap M$ and $\left(x_{0}, \partial A_{0}\right)>\delta_{0}$ (see Property 2), and $x_{m} y_{m}$ be arcs in $M$ with diam $x_{m} y_{m}<\varepsilon=$ $\delta_{0} / 2$. Then almost all arcs $h_{m}\left(x_{m} y_{m}\right)$ have diameter less than $\delta_{0}$. We claim that these arcs are contained in $A_{0}$ for, if not, there is an $\operatorname{arc} h_{m}\left(x_{m} y_{m}\right)$ of diameter less than $\delta_{0}$ with a point $z \in h_{m}\left(x_{m} y_{m}\right) \backslash A_{0}$. Then there is an arc $z p \subset h_{m}\left(x_{m} y_{m}\right)$ such that $(z p \backslash\{p\}) \cap A_{0}=\varnothing$ and $p \in A_{0}$. Since $\rho\left(x_{0}, \partial A_{0}\right)>\delta_{0}, x_{0} \in h_{m}\left(x_{m} y_{m}\right)$ and $\operatorname{diam} h_{m}\left(x_{m} y_{m}\right)<\delta_{0}$, we have $p \in A_{0} \backslash \partial A_{0}$ and the set $A_{0} \cup z p \subset M$ is an $n$-dimensional simple triod, which is impossible by Property 1 . 
Thus almost all $\operatorname{arcs} h_{m}\left(x_{m} y_{m}\right)$ are contained in $A_{0}$. By the local connectedness of $A_{0}$ there are arcs $A_{m}$ in $A_{0}$ (for sufficiently great $m$ ) such that $h_{m}\left(x_{m}\right)=x_{0} \in$ $A_{m}, h_{m}\left(y_{m}\right) \in A_{m}$ and $\lim \operatorname{diam} A_{m}=0$ (because $\left.\lim h_{m}\left(y_{m}\right)=x_{0}\right)$. Then we have $\lim \operatorname{diam} h_{m}^{-1}\left(A_{m}\right)=0$ and $x_{m}, y_{m} \in h^{-1}\left(A_{m}\right) \underset{\text { top }}{=} I$, hence $\lim d\left(x_{m}, y_{m}\right)=0<\delta$, a contradiction.

Let $C$ be the family of all arc components of $M$. Note that the function $d: C \times$ $C \rightarrow E^{1}$ yields a metric to each arc component $C \in C$. This metric is equivalent to the metric $\rho$ if and only if for each sequence $\left\{x_{m}\right\}$ of points of $C$ converging to a point $x_{0} \in C$ there is a sequence of arcs $x_{m} x_{0}$ with $x_{m} x_{0} \subset C$ such that $\lim \operatorname{diam} x_{m} x_{0}=0$.

PROPERTY 4. If the metrics $d$ and $\rho$ are equivalent on an arc component of $M$, then each arc component of $M$ is a compact manifold.

ProOF. Let the metrics $d$ and $\rho$ be equivalent on a member $C$ of $C$ and let $x \in C$. Then, by Property 1 , each set $A$ homeomorphic to $I^{n}$ such that $x \in A \backslash \partial A \subset C$ is a neighborhood of $x$ in $C$. Thus $C$ is a manifold. Suppose $C$ is not compact. However, it is locally compact, so the set $\operatorname{cl} C \backslash C$ is compact. By (14.2) in [6, p. 33] $\mathrm{cl} C$ is homogeneous (compare also Property 13 in $\left[\mathbf{1}\right.$, p. 214]). Let $C_{1}$ be another arc component of $\operatorname{cl} C$. By the homogeneity of $\operatorname{cl} C$ we have $\operatorname{cl} C=\operatorname{cl} C_{1} \subset$ $\operatorname{cl}(\operatorname{cl} C \backslash C)=\operatorname{cl} C \backslash C$, a contradiction.

\section{PROPERTY 5. The arc components of $M$ are not compact manifolds.}

ProOF. Suppose an arc component of $M$ is a compact manifold. Then by the homogeneity of $M$, for each $C \in C$ so is $C$. Each $C \in C$ separates $E^{n+1}$ into exactly two components (see Theorem (27.10) in [2, p. 235]). By Theorem 9, for a given sequence $\left\{A_{m}\right\}$ with $A_{0}, A_{1}, A_{2}, \ldots \in \mathcal{C}$ the following conditions are equivalent:

(i) $\operatorname{Lim} A_{m}=A_{0}$.

(ii) $\lim \rho\left(A_{m}, A_{0}\right)=0$.

(iii) The sequence $\left\{A_{m}\right\}$ is homeomorphically convergent to $A_{0}$, i.e., there are $\varepsilon_{m}$-homeomorphisms $h_{m}: A_{0} \rightarrow A_{m}$ with $\lim \varepsilon_{m}=0$.

Therefore by Proposition 6 we conclude that if $\operatorname{Lim} A_{m}=A_{0}$ and $p, q \in E^{n+1} \backslash A_{0}$, then the two following statements are equivalent:

(i') $A_{0}$ separates $E^{n+1}$ between $p$ and $q$.

(ii') Almost all $A_{m}$ 's separate $E^{n+1}$ between $p$ and $q$.

Consequently, if $p, q \in E^{n+1} \backslash M$, the families $S(p, q)=\left\{C \in C: C\right.$ separates $E^{n+1}$ between $p$ and $q\}$ and $C \backslash S(p, q)$ are closed in $C$ with the usual topology induced by the Hausdorff distance. The equivalence (i) $\Leftrightarrow$ (ii) implies the continuity of the natural map from $M$ to $C$, whence it follows that $C$ is connected. Thus for each pair of points $p, q \in E^{n+1} \backslash M$ the family $S(p, q)$ or its complement is empty.

Let $C$ and $C_{1}$ be distinct arc components of $M$. Again by Theorem (27.10) in $\left[2\right.$, p. 235] the set $E^{n+1} \backslash\left(C \cup C_{1}\right)$ has exactly three components. Let $T_{1}$, $T_{2}, T_{3}$ be $n$-dimensional simple triods lying in mutually distinct components of $E^{n+1} \backslash\left(C \cup C_{1}\right)$. Then by Property 1 there are points $t_{i} \in T_{i} \backslash M$ for $i \in\{1,2,3\}$. Two of them, assume $t_{1}$ and $t_{2}$, lie in the same component of $E^{n+1} \backslash C$. Clearly $C_{1}$ separates $E^{n+1}$ between $t_{1}$ and $t_{2}$ (compare Theorem 11 of [4, p. 471]). Thus $S\left(t_{1}, t_{2}\right) \neq \varnothing \neq C \backslash S\left(t_{1}, t_{2}\right)$, a contradiction. 
PROPERTY 6. If $\varepsilon>0$ is a number guaranteed by Property 3 , then for each $x \in$ $M$ there is a sequence $\left\{x_{m}\right\}$ of points lying in the arc component of $M$ containing $x$, such that $\lim x_{m}=x$ and $d\left(x, x_{m}\right) \geq \varepsilon$ for each $m$.

PROOF. By Properties 4 and 5 the metrics $\rho$ and $d$ are not equivalent on an arc component $C \in C$. Hence there are a sequence $\left\{x_{m}^{0}\right\}$ with $x_{m}^{0} \in C$ and a point $x_{0} \in C$ such that $\lim \rho\left(x_{0}, x_{m}^{0}\right)=0$ and $\lim \sup d\left(x_{0}, x_{m}^{0}\right)>0$. By the homogeneity of $M$ for each point $x$ of $M$ we may find such a sequence $\left\{x_{m}\right\}$. This sequence may be chosen such that $\lim d\left(x, x_{m}\right)=\delta>0$. By Property 3 we get $\delta \geq \varepsilon$.

PROPERTY 7. There exists a number $\varepsilon>0$ such that if $0<\delta<\varepsilon, p \in E^{n+1}$, $B_{0}$ is an arc component of the nonempty set $B(p, \delta) \cap M$ and $C_{0}$ is the arc component of $M$ containing $B_{0}$, then

(a) the set $B(p, \delta) \backslash B_{0}$ has exactly two components, and

(b) if for a component $U$ of $B(p, \delta) \backslash B_{0}$ we have $\operatorname{cl}\left(U \cap C_{0}\right) \cap B_{0} \neq \varnothing$, then no point of $B_{0}$ is accessible from $U \backslash C_{0}$, and

(c) for at least one component $U$ of $B(p, \delta) \backslash B_{0}$ no point of $B_{0}$ is accessible from $U \backslash C_{0}$.

PROOF. Let $\varepsilon_{2}>0$ be a number guaranteed by Property 2 for the number $\operatorname{diam} M$, let $\varepsilon_{3}>0$ be a number guaranteed by Property 3 , and suppose $\varepsilon=$ $\min \left\{\varepsilon_{2} / 2, \varepsilon_{3} / 2\right\}$. Assume $0<\delta<\varepsilon$.

(a) Let $x_{0} \in B_{0}$. By Property 2 there is $A \underset{\text { top }}{=} I^{n}$ such that $x_{0} \in A \subset M$ and $\rho\left(x_{0}, \partial A\right)>\varepsilon_{2}>2 \delta$. Taking into account Property 1 , observe that $B_{0}$ is also the arc component of $A \cap B(p, \delta)$ containing $x_{0}$ (if not, there would be an arc $x y \subset B_{0}$ with $x y \backslash\{y\} \subset B_{0} \backslash A$ not intersecting $\partial A$ by the previous inequalities). Thus by Proposition 3 we see $B_{0}$ separates $B(p, \delta)$ into exactly two components.

(b) Let $\left\{x_{m}\right\}$ be a sequence converging to a point $x \in B_{0}$, with $x_{m} \in U \cap C_{0}$, where $U$ is a component of $B(p, \delta) \backslash B_{0}$. For a given point $y \in B_{0}$ let $x y$ be an arc in $B_{0}$ and let $h: M \rightarrow M$ be $\varepsilon_{m}$-homeomorphisms with $\lim \varepsilon_{m}=0$ such that $h_{m}(x)=x_{m}$ (see Theorem 9). For sufficiently great $m$ the $\operatorname{arcs} h_{m}(x y)$ lie in $B(p, \delta)$, and for those $m$, we have $h_{m}(x y) \subset U \cap C_{0}$. Now we have to prove that $y$ is not accessible from $U \backslash C_{0}$. In order to do this find a set $A_{0} \underset{\text { top }}{=} I^{n}$ such that $y \in A_{0} \backslash \partial A_{0} \subset A_{0} \subset B_{0}$. Then for almost all integers $m$ we have $h_{m}\left(A_{0}\right) \subset U$. Applying Proposition 7 for the set $A$ defined in case (a) we get the conclusion.

(c) It is a corollary to (a) and (b) and to Property 6. In fact, for a given point $x \in B_{0}$ take a sequence $\left\{x_{m}\right\}$ guaranteed by Property 6 (note that $x_{m} \in C_{0} \backslash B_{0}$ ), find one of two components of $B(p, \delta) \backslash B_{0}$ with infinite number of $x_{m}$ 's (see Property 7(a)) and apply Property 7(b). This completes the proof of Property 7.

Property 7 justifies the names introduced below. Let $\delta, p, B_{0}$ and $C_{0}$ have the same meanings as in Property 7 such that the conclusions (a), (b), (c) of this property hold and let $U, V$ be distinct components of $B(p, \delta) \backslash B_{0}$. Then $U$ and $V$ are called the sides of $B_{0}$ in $B(p, \delta)$ and we say that each of them is opposite to the other. If no point of $B_{0}$ is accessible from $U \backslash C_{0}$, then $U$ is called a side of nonaccessibility of $B_{0}$ in $B(p, \delta)$.

PROPERTY 8. There exists $\varepsilon>0$ such that for every positive $\delta<\varepsilon$ there is $\xi>0$ such that if $x_{0} \in M$ and if $f: I \rightarrow C_{0}, g_{0}, g_{1}: I \rightarrow E^{n+1}$ are mappings 
(where $C_{0}$ is, as previously, the arc component of $M$ containing $x_{0}$ ) satisfying

(i) $f(0)=x_{0}$,

(ii) $\rho\left(f(t), g_{i}(t)\right)<\xi$ for $i \in\{0,1\}$ and $t \in I$,

(iii) the arc component $A_{t}^{i}$ of $g_{i}(I) \cap B(f(t), \delta)$ containing $g_{i}(t)$ for $i \in\{0,1\}$ is disjoint with the arc component $B_{t}$ of $C_{0} \cap B(f(t), \delta)$ containing $f(t)$, i.e., $A_{t}^{0} \cap B_{t}=$ $A_{t}^{1} \cap B_{t}=\varnothing$ for every $t \in I$,

then the two following implications hold:

(a) If $g_{0}(0)$ lies in a side of nonaccessibility of $B_{0}$ in $B\left(x_{0}, \delta\right)$, then for every $t \in I$ the point $g_{0}(t)$ lies in a side of nonaccessibility of $B_{t}$ in $B(f(t), \delta)$.

(b) If the points $g_{0}(0)$ and $g_{1}(0)$ lie in opposite sides of $B_{0}$ in $B\left(x_{0}, \delta\right)$, then for every $t \in I$ the points $g_{0}(t)$ and $g_{1}(t)$ lie in opposite sides of $B_{t}$ in $B(f(t), \delta)$.

PROOF. Let $\varepsilon_{2}, \varepsilon_{3}>0$ be numbers as in the proof of Property 7 and $\varepsilon_{7}>0$ be a number guaranteed by Property 7 . Put $\varepsilon=\min \left\{\varepsilon_{2} / 2, \varepsilon_{3} / 3, \varepsilon_{7}\right\}$, and let $0<\delta<\varepsilon$. Then there is an integer $k$ such that if $d(x, y)<\varepsilon_{3}$ and $\rho(x, y)<\delta / k$, then $d(x, y)<\delta$ for each points $x, y$ of the same arc component of $M$ (see Property $3)$. Let $3 \xi$ with $0<3 \xi<\delta / 4 k$ be a number guaranteed by Property 3 for the number $\delta / 4 k$, i.e. if $d(x, y)<\varepsilon_{3}$ and $\rho(x, y)<3 \xi$, then $d(x, y)<\delta / 4 k$ for $x$, $y$ in the same arc component of $M$. Assume the mappings $f, g_{0}, g_{1}$ satisfy the statements (i)-(iii). Let $\varepsilon_{0}>0$ be a number such that if $\left|t_{1}-t_{2}\right|<\varepsilon_{0}$, then

$$
\max \left\{\rho\left(f\left(t_{1}\right), f\left(t_{2}\right)\right), \rho\left(g_{0}\left(t_{1}\right), g_{0}\left(t_{2}\right)\right), \rho\left(g_{1}\left(t_{1}\right), g_{1}\left(t_{2}\right)\right)\right\}<\xi \text { for } t_{1}, t_{2} \in I \text {. }
$$

(a) Put $P=\left\{t \in I: g_{0}(t)\right.$ lies in a side of nonaccessibility of $B_{t}$ in $\left.B(f(t), \delta)\right\}$. Suppose there are $t_{0}, t \in I$ with $0<t-t_{0}<\varepsilon_{0}$ such that $t_{0} \in P$ and $t \notin P$. Put $a=g_{0}(t)$. There is a segment $\overline{a x}$ with $x \in B_{t}$ and $\rho(a, x)=\rho\left(a, B_{t}\right) \leq \rho(a, f(t))<$ $\xi$. Then we have $d\left(f\left(t_{0}\right), f(t)\right) \leq \operatorname{diam} f\left(\left[t_{0}, t\right]\right)<\xi<\varepsilon_{3}$ and $\rho\left(f\left(t_{0}\right), f(t)\right)<\xi$. Thus $d\left(f\left(t_{0}\right), f(t)\right)<\delta$ by Property 3. Therefore

$$
d\left(f\left(t_{0}\right), x\right) \leq d\left(f\left(t_{0}\right), f(t)\right)+d(f(t), x)<\delta+2 \delta<3 \varepsilon<\varepsilon_{3}
$$

and

$$
\rho\left(f\left(t_{0}\right), x\right) \leq \rho\left(f\left(t_{0}\right), f(t)\right)+\rho\left(f(t), g_{0}(t)\right)+\rho(a, x)<3 \xi .
$$

Hence, again by Property 3 we have $d\left(f\left(t_{0}\right), x\right)<\delta / 4 k<\delta$ and $x \in B_{t_{0}}$. If $t^{\prime} \in\left[t_{0}, t\right] \subset I$, then

$$
\rho\left(f\left(t_{0}\right), g_{0}\left(t^{\prime}\right)\right) \leq \rho\left(f\left(t_{0}\right), g_{0}\left(t_{0}\right)\right)+\rho\left(g_{0}\left(t_{0}\right), g_{0}\left(t^{\prime}\right)\right)<2 \xi<\delta,
$$

Thus $g_{0}\left(\left[t_{0}, t\right]\right) \subset A_{t_{0}}^{0} \subset B\left(f\left(t_{0}\right), \delta\right)$. Since $A_{t_{0}}^{0} \cap B_{t_{0}}=\varnothing$, the points $g_{0}\left(t_{0}\right), g_{0}(t)$ lie in the same side of nonaccessibility of $B_{t_{0}}$ in $B\left(f\left(t_{0}\right), \delta\right)$.

If $(\overline{a x} \backslash\{x\}) \cap B_{t_{0}}=\varnothing$, then since $x$ is not accessible from the side of $B_{t_{0}}$ containing $a=g_{0}(t)$, for every $y \in \overline{a x} \backslash\{x\}$ the set $\overline{y x} \backslash\{x\}$ intersects $C_{0}$. Hence by Property 7 (b) $g_{0}(t)$ lies in the side of nonaccessibility of $B_{t}$ in $B(f(t), \delta)$, a contradiction.

If there is $y \in(\overline{a x} \backslash\{x\}) \cap B_{t_{0}}$, then

$$
d(y, f(t)) \leq d\left(y, f\left(t_{0}\right)\right)+d\left(f\left(t_{0}\right), f(t)\right)<2 \delta+\delta<3 \varepsilon \leq \varepsilon_{3}
$$

and

$$
\rho(y, f(t)) \leq \rho\left(y, g_{0}(t)\right)+\rho\left(g_{0}(t), f(t)\right)<2 \xi<3 \xi,
$$

hence $d(f(t), y)<\delta / 4 k<\delta$ and $y \in B_{t}$, which is impossible by the construction of the segment $\overline{a x}$. 
Therefore we infer that if $t_{0} \in P$, then $t \in P$ for each $t \in\left[t_{0}, t_{0}+\varepsilon_{0}\right) \cap I$ and, consequently, that $P=I$ if $0 \in P$.

(b) Put $Z=\left\{t \in I: g_{0}(t), g_{1}(t)\right.$ lie in opposite sides of $B_{t}$ in $\left.B(f(t), \delta)\right\}$. Suppose there are $t_{0}, t \in I$ with $0<t-t_{0}<\varepsilon_{0}$ such that $t_{0} \in Z$ and $t \notin Z$. Let $0 \in Z$. By Property $7(\mathrm{c})$ at least one of the points $g_{0}(0), g_{1}(0)$ lies in a side of nonaccessibility of $B_{0}$ in $B\left(x_{0}, \delta\right)$. Applying conclusion (a) we see that $g_{0}(t)$ and $g_{1}(t)$ both lie in a side of nonaccessibility of $B_{t}$ in $B(f(t), \delta)$. Put $a=g_{0}(t), b=f(t)$ and $c=g_{1}(t)$. Consider the first point $x$ of the segment $\overline{a b}$ belonging to $B_{t}$ (in the ordering from $a$ to $b$ ) and the first point $y$ of the segment $\overline{c b}$ belonging to $B_{t}$ (in the ordering from $c$ to $b$ ). Then there is a sequence $\left\{x_{m}\right\}$ converging to $x$ with $x_{m} \in(\overline{a x} \backslash\{x\}) \cap C_{0}$. Let $h_{m}: M \rightarrow M$ be $\varepsilon_{m}$-homeomorphisms with $\lim \varepsilon_{m}=0$ and $h_{m}(x)=x_{m}$. We claim that there are an integer $j$ and a point $y_{0} \in h_{j}\left(B_{t}\right) \cap(\overline{c y} \backslash\{y\})$ such that $x_{j}$ and $y_{0}$ are points of the same arc component of $C_{0} \cap B(f(t), \delta)$. In fact, let $x y \subset B_{t}$ be an arc and $A_{0} \subset B_{t}$ be a set homeomorphic to $I^{n}$ with $y \in A_{0} \backslash \partial A_{0}$. Since $x_{m} \in h_{m}\left(A_{0} \cup x y\right)$, the sets $h_{m}\left(A_{0} \cup x y\right)$ and the point $c$ lie in the same side $U_{t}$ of $B_{t}$ in $B(f(t), \delta)$ for sufficiently great numbers $m$. By Proposition 7 it follows that $y$ is not accessible from $U_{t} \backslash\left(h_{1}\left(A_{0}\right) \cup h_{2}\left(A_{0}\right) \cup \cdots\right)$, hence there is $y_{0} \in h_{j}\left(A_{0} \cup x y\right) \cap(\overline{c y} \backslash\{y\}) \subset B(f(t), \delta) \cap h_{j}\left(B_{t}\right) \cap(\overline{c y} \backslash\{y\})$ for some integer $j$, so the claim is obtained. Therefore $d\left(x_{j}, y_{0}\right)<2 \delta<2 \varepsilon \leq \varepsilon_{3}$. We also have

$$
\rho\left(x_{j}, y_{0}\right) \leq \rho\left(x_{j}, b\right)+\rho\left(b, y_{0}\right) \leq \rho(a, b)+\rho(b, c)<2 \xi<3 \xi,
$$

hence $d\left(x_{j}, y_{0}\right)<\delta / 4 k$. Let $A \subset C_{0}$ be an arc with $x_{j}, y_{0} \in A$ and $\operatorname{diam} A<\delta / 4 k$. Consider the union $U=\overline{a x}_{j} \cup A \cup \overline{y_{0} c}$. Note that $U \subset \bigcap\left\{B\left(f\left(t^{\prime}\right), \delta\right): t_{0} \leq t^{\prime} \leq t\right\}$. Indeed, if $u \in U$, then

$$
\rho(f(t), u) \leq \rho(f(t), a)+\rho\left(a, x_{j}\right)+\operatorname{diam} A+\rho\left(y_{0}, c\right) \leq 3 \xi+\delta / 4 k,
$$

and for $t^{\prime} \in\left[t_{0}, t\right]$

$$
\rho\left(f\left(t^{\prime}\right), u\right) \leq \rho\left(f\left(t^{\prime}\right), f(t)\right)+\rho(f(t), u) \leq \xi+3 \xi+\delta / 4 k<2 \delta / 4 k+\delta / 4 k<\delta
$$

(recall that $3 \xi<\delta / 4 k$ ).

By the construction of $U$ we conclude that $U \cap B_{t}=\varnothing$. Suppose there is a point $x \in U \cap B_{t^{\prime}}$ for some $t^{\prime} \in\left[t_{0}, t\right]$. Then

$$
d(z, f(t)) \leq d\left(z, f\left(t^{\prime}\right)\right)+d\left(f\left(t^{\prime}\right), f(t)\right)<2 \delta+\xi<3 \delta<3 \varepsilon<\varepsilon_{3}
$$

and

$$
\rho(z, f(t)) \leq 3 \xi+\delta / 4 k \leq 2 \delta / 4 k<\delta / k,
$$

hence $d(z, f(t))<\delta$ and $z \in U \cap B_{t} \neq \varnothing$ which contradicts the previous conclusion. Therefore we have $U \cap B_{t^{\prime}}=\varnothing$ for each $t^{\prime} \in\left[t_{0}, t\right]$. If $t^{\prime} \in\left[t_{0}, t\right]$, for $i \in\{0,1\}$ we also get

$$
\rho\left(g_{i}\left(t^{\prime}\right), f\left(t_{0}\right)\right) \leq \rho\left(g_{i}\left(t^{\prime}\right), f\left(t^{\prime}\right)\right)+\rho\left(f\left(t^{\prime}\right), f\left(t_{0}\right)\right)<2 \xi<\delta,
$$

and so $g_{i}\left(\left[t_{0}, t\right]\right) \subset B\left(f\left(t_{0}\right), \delta\right)$. Note that the set $V=g_{0}\left(\left[t_{0}, t\right]\right) \cup U \cup g_{1}\left(\left[t_{0}, t\right]\right)$ is connected, $g_{0}\left(t_{0}\right), g_{1}\left(t_{0}\right) \in V \subset B\left(f\left(t_{0}\right), \delta\right)$ and $V \cap B_{t_{0}}=\varnothing$ (see assumption (iii)). Thus $g_{0}\left(t_{0}\right)$ and $g_{1}\left(t_{0}\right)$ lie in the same side of $B_{t_{0}}$ in $B\left(f\left(t_{0}\right), \delta\right)$, a contradiction. Similarly as in the proof of conclusion (a) for the set $P$, here we infer that $Z=I$. The proof is complete.

Fix a number $\varepsilon_{7}>0$ guaranteed by Property 7. For every positive number $\delta<\varepsilon_{7}$ and every $x \in M$ we define a number $p_{\delta}(x)$ as follows. Let $C$ be the arc component 
of $M$ containing $x$ and $C_{\delta}(x)$ be the arc component of $C \cap B(x, \delta)$ containing $x$. Then by Property 7 the set $B(x, \delta) \backslash C_{\delta}(x)$ has exactly two components: $V_{\delta}^{1}(x)$ and $V_{\delta}^{2}(x)$. Define two numbers

$$
p_{\delta}^{i}(x)= \begin{cases}\rho\left(x, V_{\delta}^{i}(x) \cap C\right), & \text { if } V_{\delta}^{i}(x) \cap C \neq \varnothing, \\ \delta & \text { otherwise, }\end{cases}
$$

for $i \in\{1,2\}$, and put $p_{\delta}(x)=\max \left\{p_{\delta}^{1}(x), p_{\delta}^{2}(x)\right\}$.

Using Property 7 (b) the reader may easily verify that

$$
\text { the condition } p_{\delta}(x)=0 \text { is equivalent to the property }
$$$$
\text { that no point of } C_{\delta}(x) \text { is accessible from } E^{n+1} \backslash C \text {. }
$$

This conclusion gives us an implication

$$
\text { if } p_{\delta}(x)=0 \text { and } 0<\xi<\delta \text {, then } p_{\xi}(x)=0 \text {. }
$$

PROPERTY 9. There exists $\varepsilon>0$ such that for each arc component $C$ of $M$ if $0<\delta<\varepsilon$, then $\inf \left\{p_{\delta}(x): x \in C\right\}=0$.

PROOF. Let $\varepsilon_{3}$ be a number guaranteed by Property 3 and $\delta_{2}$ be a number guaranteed by Property 2 for the number $\varepsilon_{3} / 4$. Put $\varepsilon=\delta_{2}$ and let $0<\delta<\varepsilon$. Fix any arc component $C \in C$, any point $x \in C$ and some numbers $\delta_{0}, \delta_{1}$ with $\delta<\delta_{0}<\delta_{1}<\varepsilon$. Then there is a sequence of points $x_{m} \in C$ converging to $x$ with $d\left(x, x_{m}\right) \geq \varepsilon_{3}$ (see Property 6). Thus $x_{m} \in C \backslash C_{\delta}(x)$.

If $p_{\delta_{1}}(x)=0$ then by $(* *)$ we have $p_{\delta}(x)=0$ and the conclusion holds.

Let $p_{\delta_{1}}(x)=\psi>0$ and let $\varphi>0$. We will find a point $x_{j} \in C$ such that $p_{\delta}\left(x_{j}\right)<\varphi$. There is $k \in\{1,2\}$ such that $C \cap V_{\delta_{1}}^{k}(x) \cap B(x, \psi)=\varnothing$. Assume $k=1$. Then we may find a point $y \in V_{\delta_{1}}^{1}(x) \backslash M$ and an arc

$$
y x \subset\left(V_{\delta_{1}}^{1}(x) \cup C_{\delta_{1}}(x)\right) \cap B(x, \psi) \cap B(x, \delta / 2) .
$$

Denote by $\xi$ a number guaranteed by Property 3 for the number $\delta$ (i.e. if $a, b \in$ $C^{\prime} \in C, d(a, b)<\varepsilon_{3}$ and $\rho(a, b)<\xi$, then $\left.d(a, b)<\delta\right)$. Then for some $x_{i} \in V_{\delta_{1}}^{2}(x)$ we have

$$
\rho\left(x, x_{i}\right)<\min \left\{\xi / 3, \delta / 2, \varphi / 2, \delta_{0}-\delta\right\}
$$

The set $C_{\delta_{1}}(x)$ is contained in a set $A \subset B\left(x, \varepsilon_{3} / 4\right) \cap M$ homeomorphic to $I^{n}$ such that $\rho(x, \partial A)>\varepsilon$ (see Properties 2 and 1). Thus $\operatorname{diam} A<\varepsilon_{3} / 2$ and since the set $C_{\delta_{1}}(x)$ separates $B\left(x, \delta_{1}\right)$ between $y$ and $x_{i}$, the set $A$ does the same. Consider $\varepsilon_{m}$-homeomorphisms $h_{m}: M \rightarrow M$ such that $h_{m}(x)=x_{m}$ and $\lim \varepsilon_{m}=0$ (see Theorem 9 ) and take the images $h_{m}(A)$. By Proposition 6 there is an integer $j$ such that $h_{j}(A)$ separates $B\left(x, \delta_{0}\right)$ between $y$ and $x_{i}$, diam $h_{j}(A)<\varepsilon_{3} / 2, \rho\left(x_{j}, \partial h_{j}(A)\right)>\varepsilon$, $x_{j} \in V_{\delta_{1}}^{2}(x)$ and $\rho\left(x, x_{j}\right)<\rho\left(x, x_{i}\right)$. Observe that $A \cap h_{j}(A)=\varnothing$. Indeed, it is a consequence of inequalities $d\left(x, x_{j}\right) \geq \varepsilon_{3}>\operatorname{diam} A+\operatorname{diam} h_{j}(A)$. For each $z \in y x$ we have

$$
\rho\left(z, x_{j}\right) \leq \rho(z, x)+\rho\left(x, x_{j}\right) \leq \rho(z, x)+\rho\left(x, x_{i}\right)<2 \delta / 2=\delta,
$$

hence $y x \subset B\left(x_{j}, \delta\right)$. We can prove in a similar manner that $\rho\left(x_{i}, x_{j}\right)<\delta$. Since $\left(\left(V_{\delta_{1}}^{1}(x) \cap B(x, \psi)\right) \cup C_{\delta_{1}}(x)\right) \cap\left(C \backslash C_{\delta_{1}}(x)\right)=\varnothing, y x \subset\left(V_{\delta_{1}}^{1}(x) \cap B(x, \psi)\right) \cup C_{\delta_{1}}(x)$ and $h_{j}(A) \subset C \backslash C_{\delta_{1}}(x)$, we have $y x \cap h_{j}(A)=\varnothing$ and $h_{j}(A)$ separates $B\left(x, \delta_{0}\right)$ between $x$ and $x_{i}$. The set $h_{j}(A)$ also separates the ball $B\left(x_{j}, \delta\right) \subset B\left(x, \delta_{0}\right)$ between $x$ and 
$x_{i}$. By Proposition 4 at least one arc component $B$ of $h_{j}(A) \cap B\left(x_{j}, \delta\right)$ does the same. Let $y_{0} \in \overline{x x}_{i} \cap B$. Then $d\left(y_{0}, x_{j}\right) \leq \operatorname{diam} h_{j}(A)<\varepsilon_{3}$ and

$$
\rho\left(y_{0}, x_{j}\right) \leq \rho\left(y_{0}, x_{i}\right)+\rho\left(x_{i}, x_{j}\right)<\rho\left(x, x_{i}\right)+\rho\left(x_{i}, x\right)+\rho\left(x, x_{j}\right)<3 \cdot \xi / 3=\xi ;
$$

thus $d\left(y_{0}, x_{j}\right)<\delta$. Since $\rho\left(x_{j}, \partial h_{j}(A)\right)>\varepsilon>\delta$, we have $C_{\delta}\left(x_{j}\right) \subset h_{j}(A)$ (otherwise we would get an $n$-dimensional simple triod in $M)$. Consequently, $C_{\delta}\left(x_{j}\right) \subset B$ because $y_{0} \in B \cap C_{\delta}\left(x_{j}\right) \neq \varnothing$. The inverse inclusion is obvious. Thus $B=C_{\delta}\left(x_{j}\right)$. Hence the points $x$ and $x_{i}$ lie in opposite sides of $C_{\delta}\left(x_{j}\right)$ in $B\left(x_{j}, \delta\right)$. This implies

$$
p_{\delta}\left(x_{j}\right) \leq \max \left\{\rho\left(x_{j}, x\right), \rho\left(x_{j}, x_{i}\right)\right\} \leq \max \left\{\rho\left(x_{j}, x\right), 2 \cdot \rho\left(x_{i}, x\right)\right\}=2 \cdot \rho\left(x_{i}, x\right)<\varphi,
$$

which completes the proof.

PROPERTY 10. No point of $M$ is accessible from $E^{n+1} \backslash M$.

ProOF. Let $x \in M$ and $C$ be the arc component of $M$ containing $x$. We will show that there is $\delta>0$ such that $p_{\delta}(x)=0$, which will imply the conclusion by $(*)$.

Let $\varepsilon_{i}$ be a number guaranteed by Property $i$ for $i \in\{3,7,8,9\}$. Put $\delta=$ $\min \left\{\varepsilon_{3}, \varepsilon_{7}, \varepsilon_{8}, \varepsilon_{9}\right\} / 5$. For a given positive number $\sigma<\delta$ let $\xi<\sigma$ be a number guaranteed by Property 8 for the number $\delta, \tau<\xi$ be a number guaranteed by Property 3 for the number $\xi$ and $\varphi<\tau$ be a number guaranteed by Theorem 9 for the number $\tau$. By Property 9 there is $y \in C$ with $p_{\delta}(y)<\varphi$, i.e. there are $v_{1}$, $v_{2} \in C$ lying in opposite sides of $C_{\delta}(y)$ in $B(y, \delta)$ with $\max \left\{\rho\left(y, v_{1}\right), \rho\left(y, v_{2}\right)\right\}<\varphi$. Let $f: I \rightarrow C$ be an embedding such that $f(0)=y$ and $f(1)=x$. By Theorem 9 there are $\tau$-homeomorphisms $h_{1}, h_{2}: M \rightarrow M$ such that $h_{1}(y)=v_{1}$ and $h_{2}(y)=v_{2}$. Put $g_{i}(t)=h_{i} f(t)$ for $t \in I$ and $i \in\{1,2\}$. Thus we have $\rho\left(f(t), g_{i}(t)\right)<\tau$ for all $t \in I$ and $i \in\{1,2\}$. Put $B_{t}=C_{\delta}(f(t))$ and let $A_{t}^{i}$ be the arc component of $B(f(t), \delta) \cap g_{i}(I)$ containing $g_{i}(t)$.

Suppose $z \in B_{t_{0}} \cap A_{t_{0}}^{i}$. Then

$$
\begin{aligned}
d\left(f\left(t_{0}\right), g_{i}\left(t_{0}\right)\right) & \leq d\left(f\left(t_{0}\right), z\right)+d\left(z, g_{i}\left(t_{0}\right)\right) \leq \operatorname{diam} B_{t_{0}}+\operatorname{diam} A_{t_{0}}^{i} \\
& \leq 2 \delta+2 \delta \leq 4 \varepsilon_{3} / 5<\varepsilon_{3}
\end{aligned}
$$

and $\rho\left(f\left(t_{0}\right), g_{i}\left(t_{0}\right)\right)<\tau$. Thus $d\left(f\left(t_{0}\right), g_{i}\left(t_{0}\right)\right)<\xi<\delta$. Let $\psi>0$ be a number such that if $\left|t_{1}-t_{2}\right|<\psi$, then

$$
\max \left\{\rho\left(f\left(t_{1}\right), f\left(t_{2}\right)\right), \rho\left(g_{i}\left(t_{1}\right), g_{i}\left(t_{2}\right)\right)\right\}<\delta \quad \text { for } t_{1}, t_{2} \in I \text { and } i \in\{1,2\} .
$$

Then the conditions $d\left(f\left(t_{1}\right), g_{i}\left(t_{1}\right)\right)<\delta$ and $\left|t_{1}-t_{2}\right|<\psi$ imply that

$$
d\left(f\left(t_{2}\right), g_{i}\left(t_{2}\right)\right) \leq d\left(f\left(t_{2}\right), f\left(t_{1}\right)\right)+d\left(f\left(t_{1}\right), g_{i}\left(t_{1}\right)\right)+d\left(g_{i}\left(t_{1}\right), g_{i}\left(t_{2}\right)\right)<3 \delta<\varepsilon_{3}
$$

and since $\rho\left(f\left(t_{2}\right), g_{i}\left(t_{2}\right)\right)<\tau$, thus by Property 3 we have $d\left(f\left(t_{2}\right), g_{i}\left(t_{2}\right)\right)<\xi<\delta$. Applying these implications finitely many times (if $t$ runs from $t_{0}$ to 0 ) we get $d\left(f(0), g_{i}(0)\right)=d\left(y, v_{i}\right)<\delta$, a contradiction.

Therefore we have $B_{t} \cap A_{t}^{1}=B_{t} \cap A_{t}^{2}=\varnothing$ for each $t \in I$. Recall that for each $t \in I$ and each $i \in\{1,2\}$ we have $\rho\left(f(t), g_{i}(t)\right)<\tau<\xi$. Applying Property 8 we see that the points $g_{1}(1), g_{2}(1) \in C$ lie in opposite sides of $C_{\delta}(x)$ in $B(x, \delta)$ and thus $p_{\delta}(x)<\xi<\sigma$. Hence $p_{\delta}(x)=0$.

To make the proof of the Main Theorem complete, note at least an obvious property of $M$ (being true for each nonempty compact subset of $E^{n+1}$ ) which contradicts the previous one.

PROPERTY 11. There exists a point in $M$ that is accessible from $E^{n+1} \backslash M$. 


\section{REFERENCES}

1. R. H. Bing, A simple closed curve is the only homogeneous bounded plane continuum that contains an arc, Canad. J. Math. 12 (1960), 209-230.

2. M. J. Greenberg and J. R. Harper, Algebraic topology, a first course, Benjamin/Cummings, Reading, Mass., 1981.

3. C. L. Hagopian, Homogeneous plane continua, Houston J. Math. 1 (1975), 35-41.

4. K. Kuratowski, Topology. II, PWN, Warszawa, 1968.

5. A. Lelek, On the Moore triodic theorem, Bull. Acad. Polon. Sci. Sér. Sci. Math. Astronom. Phys. 8 (1960), 271-276.

6. T. Maćkowiak and E. D. Tymchatyn, Continuous mappings on continua. II, Dissertationes Math. 225(1984), 1-57.

7. S. Mazurkiewicz, Sur les continus homogenes, Fund. Math. 5 (1924), 137-146.

Institute of Mathematics, Opole Pedagogical University, 45-951 Opole, Ul. OLESKa 48, POLAND 